Bundesgesundheitsbl 2011 · 54:905-906

DOI 10.1007/s00103-011-1322-1

Online publiziert: 26. Juli 2011

(c) Springer-Verlag 2011

\title{
S.N. Willich
}

Institut für Sozialmedizin, Epidemiologie und Gesundheitsökonomie, Charité - Universitätsmedizin Berlin, Berlin

\section{Die Neuen Alten}

\section{Versorgungsforschung für eine veränderte Gesellschaft}

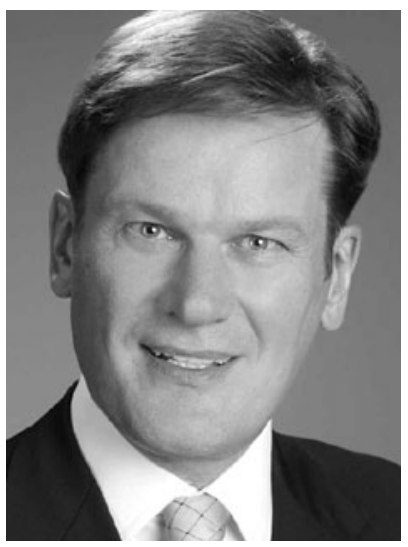

nen Zeit, erhöht sich seit rund 160 Jahren jährlich um drei Monate pro Jahr, und die Hälfte der heute geborenen Kinder wird mindestens 102 Jahre alt werden. Dabei zeigt sich in einigen Ländern ein ungebrochen starker weiterer Anstieg, in anderen Ländern wiederum stagniert der weitere Zuwachs. Insbesondere für die Finanzierbarkeit der Gesundheitssysteme ist die Frage höchst relevant, ob es eine biologische Limitation der Lebenserwartung gibt, die wir früher oder später erreichen.

Die befürchtete Zunahme der Morbidität im Zusammenhang mit einer steigenden Lebenserwartung lässt sich allerdings nicht grundsätzlich belegen. Die zunehmende Prävalenz der wichtigen chronischen Erkrankungen wie Herz-KreislaufErkrankungen, Diabetes oder Krebs ist im Wesentlichen auf frühere Diagnostik und effizientere Behandlung zurückzuführen, demzufolge die Sterblichkeit geringer wird als auf die Anzahl der Neuerkrankungen. Mit zunehmender Lebenserwartung steigt nach heutigen Erkenntnissen somit auch die Zahl der Lebensjahre, die ohne Beeinträchtigung der Aktivitäten des täglichen Lebens und funktionelle Beeinträchtigungen gelebt werden.

Prof. Dr. Adelheid Kuhlmey aus dem Institut für Medizinische Soziologie der Charité - Universitätsmedizin Berlin und ausgewiesene Expertin auf dem Gebiet der Versorgungsforschung im Alter befasst sich mit der Frage, inwieweit sich die Versorgungsnotwendigkeiten gewandelt haben und welchen Beitrag die Forschung zur Anpassung des Gesundheitssystems leisten kann. Die zunehmende Prävalenz chronischer Erkrankungen führt zu einer deutlichen Zunahme der Multimorbidität, die dann im hochbetagten Alter ein hohes Risiko von Hilfeabhängigkeit und Pflegebedürftigkeit birgt. Im Jahr 2050 werden 4,35 Mio. Pflegebedürftige in Deutschland vorausgesagt. Ein weiterer Risikofaktor für Pflegebedürftigkeit ist die Demenz, an der schon jetzt $7 \%$ der über 65-Jährigen leiden. Diesem komplexen Versorgungsbedarf alter Menschen wird das Gesundheitssystem wegen der mangelnden Kontinuität und Vernetzung der Versorgung sowie der unzulänglichen ambulanten Infrastruktur nicht gerecht. Im Falle eines komplexen Hilfebedarfs sollte der Versorgungsmix aus professioneller und privater Betreuung entwickelt werden. Kuhlmey fordert hier ein Umdenken professioneller Versorger hin zu einer kooperativen Ge- 
sundheitsversorgung zusammen mit privater und ehrenamtlicher Betreuung.

Prof. Dr. Ingo Füsgen vom Lehrstuhl für Geriatrie an der Universität Witten/ Herdecke beschreibt in seinem Beitrag Modelle der integrierten geriatrischen Versorgung aus der Sicht des praktisch klinisch Tätigen. Füsgen fordert eine breiter angelegte geriatrische Aus-, Fort- und Weiterbildung von professionellen und Laienhelfern. Er kritisiert das in der geltenden Approbationsordnung angelegte Querschnittsfach „Medizin des Alterns“ als zu wenig durch spezialisierte Geriater getragen. Die Gesundheitsversorgung alter Menschen verlangt umfassenderes Vorgehen bereits bei der Diagnostik: Neben der üblichen Anamnese und Befunderhebung muss es ein geriatrisches Assessment geben, welches Fähigkeiten und Ansprüche alter Menschen spezifisch erfasst. Während der Betreuung - sei es im ambulanten oder stationären Sektor - ist die Mitarbeit oder Beratung durch ein geriatrisches Team in allen Fachbereichen erforderlich. Die Gesundheitsversorgung muss personen- und bedarfsorientiert sein, das kann im Einzelfall auch heißen, statt professioneller Hilfe andere Lösungen zu suchen. Die Grenzen zwischen stationärem und ambulantem Sektor müssen eingerissen werden, um abgestufte Angebote wie teilstationäre oder mobile ambulante Dienste zu ermöglichen. Die Grenzen der medizinischen Fachgebiete müssen ebenso aufgeweicht werden, denn alte Menschen benötigen Hilfe zu fachübergreifenden, syndromorientierten Problemen wie Schmerz, Sturz oder Schwindel.

Dr. Frank Berner, Leiter der Geschäftsstelle der Sechsten Altenberichtskommission am Deutschen Zentrum für Altersfragen stellt ausgewählte Aspekte aus dem Sechsten Altenbericht „Altersbilder in der Gesellschaft" vor. Der Altenbericht analysiert unter anderem die Bedeutung von Altersbildern für das Gesundheitswesen. In den letzten Jahrzehnten hat sich eine neue Lebensphase der sogenannten jungen Alten etabliert, die durch bessere soziale Lage und Gesundheitsversorgung ermöglicht wird und dem traditionell als Lebensabend bezeichneten Abschnitt vorangestellt ist. Berner betont, dass das chronologische Alter dabei als alleinige Bezugs- größe zur Definition von Lebensumständen und gesetzlichen Altersgrenzen nicht mehr tauglich ist. Die Lebensumstände sind vielfältig und entsprechend vielfältig die Bedarfslagen. Per se von Fürsorge- und Hilfebedürftigkeit bei alten Menschen auszugehen ist einseitig und sollte durch eine an den Stärken und Gestaltungsspielräumen orientierte Perspektive auf alte Menschen ergänzt werden. Daraus leitet der Altenbericht Empfehlungen ab, die Entwicklungspotenziale älterer Menschen anzuerkennen und durch geeignete Rahmenbedingungen zu ermöglichen. Gerade im Gesundheitswesen werden ältere Menschen einseitig als altersbedingt und unvermeidbar gesundheitlich beeinträchtigt angesehen und in ein negatives Altersselbstbild und Altersfremdbild gefasst. In der Folge werden die Ressourcen im Gesundheitswesen gar nicht gemäß einem positiven Altersbild, welches an den Kompetenzen der Alten orientiert ist, verteilt.

Die Autoren kommen aus unterschiedlichen Perspektiven zu wesentlichen gemeinsamen Forderungen: Krankheiten bei alten Menschen dürfen nicht vereinfacht als Alterserscheinungen angesehen werden. Die Gesundheitsversorgung, aufbauend auf passender Struktur und spezifischem Ausbildungsstand der Leistungsanbieter, muss derart gestaltet werden, dass sie das Potenzial der alten Menschen entfalten hilft, und muss an ihren ganz spezifischen Bedarfen ausgerichtet werden. Ideal und nachhaltig ist ein Mix aus professionellen Helfern, Ehrenamtlichen und Laien. Vor diesem Hintergrund hoffen wir, dass das Schwerpunktheft für Sie interessante und hilfreiche neue Aspekte darstellt.

Ihr

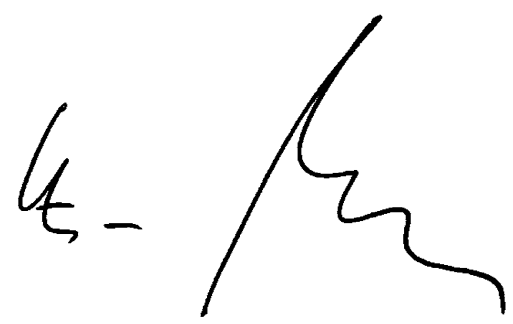

Stefan N. Willich

\section{Korrespondenzadresse}

\section{Prof. Dr. S.N. Willich}

Institut für Sozialmedizin,

Epidemiologie und Gesundheitsökonomie, Charité - Universitätsmedizin Berlin 10098 Berlin

stefan.willich@charite.de 